\title{
INCORPORAÇÃO DOS PROCESSOS DE CONHECIMENTO NAS ROTINAS DE TRABALHO ORGANIZACIONAIS: UM MODELO DE APLICAÇÃO FUNDAMENTADO NA INTEGRAÇÃO ENTRE GESTÃO DO CONHECIMENTO E BUSINESS PROCESS MANAGEMENT
}

\author{
Marco Antonio Cardoso Sena ${ }^{1}$ \\ Lia Caetano Bastos ${ }^{2}$ \\ Gertrudes Aparecida Dandolini ${ }^{3}$
}

\begin{abstract}
${ }^{1}$ Gerente de Projetos da Empresa Brasileira de Correios e Telégrafos - ECT e Doutorando do Departamento de Engenharia e Gestão do Conhecimento, Universidade Federal de Santa Catarina: Centro Tecnológico (CTC) - Campus Universitário Reitor João David Ferreira Lima. CEP: 88040-970. Trindade: Florianópolis-SC. E-mail: marcoacsena@gmail.com.

${ }^{2}$ Professora Doutora do Departamento de Engenharia e Gestão do Conhecimento, Universidade Federal de Santa Catarina: Centro Tecnológico (CTC) - Campus Universitário Reitor João David Ferreira Lima. CEP: 88040-970. Trindade: Florianópolis-SC. E-mail: liacbastos@gmail.com.

${ }^{3}$ Professora Doutora do Departamento de Engenharia e Gestão do Conhecimento, Universidade Federal de Santa Catarina: Centro Tecnológico (CTC) - Campus Universitário Reitor João David Ferreira Lima. CEP: 88040-970. Trindade: Florianópolis-SC. E-mail: ggtude@gmail.com.
\end{abstract}

\section{Recebido em: 30/05/2014 - Aprovado em: 15/09/2014 - Disponibilizado em: 15/12/2014}

\begin{abstract}
RESUMO: Não obstante ao grande número de modelos de gestão do conhecimento encontrados na literatura, vários programas nessa área, na última década, não entregaram os benefícios esperados pelas organizações, devido à desconexão entre os processos de conhecimento (identificar, criar, compartilhar, aplicar e avaliar) e o que constitui as atividades críticas à consecução dos objetivos de negócio e estratégicos. Por outro lado, estudos apontam que é crescente a orientação por processos de negócio nos ambientes corporativos, tanto de organizações privadas como públicas. Essa tendência na área de gestão deve-se ao fato de ser por meio dos processos de negócio que de fato as organizações concebem, desenvolvem e entregam produtos e serviços a clientes. Logo, para esses modelos organizacionais tendem a convergir as atividades, o conhecimento e todos os recursos críticos para os negócios de uma organização. Dessa forma, é cada vez maior a adoção do gerenciamento de processos de negócio como princípio de gestão. Nesse sentido, este artigo propõe-se a construir um modelo de incorporação dos processos de conhecimento nas rotinas de trabalho organizacionais, com base na integração entre gestão do conhecimento e gerenciamento de processos de negócio. Para isso, formularam-se proposições de pesquisas que foram investigadas por meio de um estudo de caso único, complementado por entrevistas com gestores e especialistas envolvidos com processos de negócio, no âmbito de uma empresa pública de grande porte. Os resultados contribuem com outras pesquisas no sentido de que a integração entre GC e BPM pode proporcionar várias facilidades com relação aos processos de conhecimento.
\end{abstract}

Palavras-chave: Gestão do Conhecimento. Gerenciamento de Processos de Negócio. Integração. Negócio. Modelo.

\begin{abstract}
Despite myriad knowledge management frameworks in the literature in the last decade several programs in this area did not deliver expected benefits by organizations, due to the disconnections between knowledge processes (identify, create, share, apply and evaluate) and what is critical to the activities to achievement of business and strategic goals. On the other hand, studies show that there is a growing orientation business processes in enterprise environments, both private and public organizations. This trend in management is due to the fact that through the business processes that in fact organizations design, develop and deliver products and services to customers. Therefore, for these organizational models converges the activities, knowledge and all critical resources to the business of an organization. Thus, it is increasingly adopting the management of business processes as a management principle. In this sense, this paper proposes to build a model for incorporating the knowledge processes in organizational job routines, based on the integration between knowledge management and business process management. To this end, research proposals were formulated that have been investigated by means of a single case study, supplemented by interviews with managers and specialists involved with business processes within a large public company. The results contribute to earlier searches in the sense that the integration between knowledge management and business process management can provide several facilities related to knowledge processes.
\end{abstract}

Keywords: Knowledge Management. Business Processes Management. Integration. Business. Framework. 


\section{Introdução}

A pesar de a literatura científica apresentar uma miríade de modelos de gestão do conhecimento (GC), as organizações ainda encontraram dificuldades em identificar, criar e gerir o conhecimento que é crítico para a consecução dos objetivos de negócio e estratégicos.

Por outro lado, pesquisas mostram que é crescente a adoção da orientação por processos de negócio nos ambientes corporativos, tanto do setor privado como do público conforme Houy, Fettke e Loos (2010), e a explicação para essa tendência na área da gestão organizacional se dá pelas razões a seguir, entre outras: (i) é por meio dos processos de negócio que as organizações de fato concebem, desenvolvem e entregam produtos e serviços a clientes, (ii) os processos de negócio são considerados o contexto prático para a criação e aplicação de conhecimento e (iii) os processos de negócio são os modelos organizacionais por meio dos quais as organizações podem inovar e criar valor para mercados.

Do que precede, este artigo propõe um modelo de incorporação dos processos de conhecimento (identificar, criar, compartilhar, aplicar e avaliar) nas rotinas de trabalho organizacionais, fundamentado na integração entre GC e gerenciamento de processos de negócio (Business Process Management BPM), com o objetivo de facilitar e promover a gestão do conhecimento crítico à consecução dos objetivos de negócio e estratégicos, particularmente no âmbito de empresas públicas.

\section{Planejamento da Pesquisa}

A Figura 1 apresenta a classificação da pesquisa que foi empreendida para concretizar o objetivo deste artigo, baseando-se nas orientações de Marconi e Lakatos (2003), Seidel (2007), Ventura (2007), Fratantonio (2008), Martins (2008), Gil (2010) e Yin (2010).

Figura 1 - Classificação da Pesquisa.

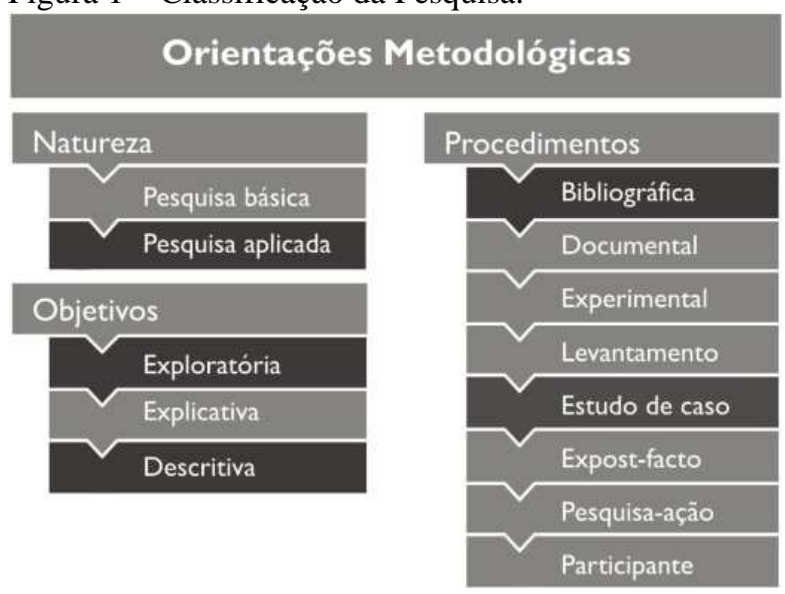

Fonte: (SOUZA, 2011).

Quanto à natureza, este trabalho caracteriza-se como aplicado, visto que está direcionado a gerar conhecimento prático visando a contribuir com a evolução do trabalho de Jung, Choi e Sung (2007), dedicado a investigar as potencialidades da integração entre GC e BPM.

No que tange aos objetivos, a pesquisa caracteriza-se como exploratória e descritiva. A abordagem exploratória é necessária uma vez que, não obstante existirem várias iniciativas que se propõem a estudar a aplicabilidade da integração entre GC e BPM, 
são escassos no Brasil trabalhos acadêmicos que têm essa natureza de investigação, em especial, no contexto das empresas públicas.

O caráter descritivo configura-se como necessário a partir do momento em que esta pesquisa visa a investigar e descrever por que e como os processos de identificação, criação, compartilhamento, aplicação e avaliação de conhecimento podem ser promovidos e, ao mesmo tempo, facilitados, por meio da integração entre GC e BPM no contexto da Empresa Brasileira de Correios e Telégrafos ECT, que é uma organização pública de grande porte.

Com relação aos procedimentos (delineamento) da pesquisa, que envolve o ambiente a ser pesquisado, o método de coleta de dados e as respectivas técnicas de análise, o trabalho objeto deste artigo delimita-se como bibliográfico, pois fundamenta-se em uma revisão da literatura sobre o tema integração entre GC e BPM, e como estudo de caso, do tipo único, que será complementado por meio de entrevistas com gestores e especialistas envolvidos com o processo de negócio e-Carta da ECT.

\section{Descrição do Caso Serviço e-Carta}

Para construir o modelo de incorporação dos processos de conhecimento nas rotinas de trabalho organizacionais, este artigo empreendeu um estudo de caso na ECT, em que o processo de negócio e-Carta, que criou um serviço híbrido (composto de componentes digitais e físicos) de mesmo nome, é a unidade de pesquisa deste artigo. A próxima seção descreve o estudo de caso realizado.

\subsection{Contextualização}

De acordo com os relatórios dos dois últimos congressos internacionais realizados em 2008 e 2012 da Universal Postal UnionUPU, organização que congrega quase duzentos operadores postais no mundo, o mercado postal global está passando por profundas mudanças, como resultado de várias tendências. Uma delas está relacionada ao uso intensivo da tecnologia da informação e comunicação - TIC, que está modificando a forma como pessoas, empresas e governos se comunicam, realizam transações comerciais e se comportam.

No âmbito do mercado postal brasileiro, a onipresença da TIC está provocando a desmaterialização das comunicações comerciais, o que é uma das características da emergente economia digital brasileira. Por conta disso, as empresas têm demandado de forma crescente o uso de soluções híbridas (digital/física) para atender as suas necessidades de comunicação.

Esse é um grande desafio para a ECT, pois o desenvolvimento de soluções de comunicação híbridas demanda a inovação de serviços postais convencionais existentes. E a inovação, por usa vez, pressupõe a criação, aplicação e gestão de conhecimento. 


\subsection{A Orientação Estratégica}

Diante desse cenário de transformações, a ECT realizou uma ampla prospecção e análise do ambiente de negócios no qual ela está e estará inserida. Esses trabalhos foram os principais insumos para a ECT desenvolver um plano estratégico aderente à atual dinâmica das economias mundial e brasileira. Um dos objetivos de negócio do novo plano estratégico da ECT é desenvolver serviços digitais e híbridos $(e-$ Services) para atender às demandas da emergente economia digital brasileira.

No início de 2010, a ECT decidiu formar um grupo de trabalho - GT para criar e implantar o serviço híbrido e-Carta. Trata-se de um serviço de comunicação empresarial, sustentado por uma plataforma postal baseada em tecnológica digital.

A partir dessa decisão, foram identificados especialistas de várias áreas de conhecimento da ECT. Entre elas: negócio, marketing, projeto, tecnologia, operacional e gestão. Uma das decisões mais importantes do GT, e que hoje se sabe representou o principal fator crítico de sucesso, foi definir que o trabalho que levaria à solução inovadora (serviço e-Carta) que os clientes da ECT desejavam na área de comunicação empresarial seria empreendido por meio da orientação por processo.

A ECT iniciara em meados de 2009 a modelagem dos principais processos de negócio da organização. Dentro dessa perspectiva, o GT analisou o plano estratégico e percebeu que a orientação por processos era um dos mais importantes direcionadores da ECT, definida em um dos objetivos estratégico da empresa.

\subsection{O Cenário de Pesquisa}

Dessa forma, o GT começou a identificar os processos existentes que sustentavam a linha de negócio comunicação. Com os seguintes direcionadores de negócio e estratégico (i) desenvolver serviços digitais e híbridos e (ii) a orientação por processos, respectivamente, o GT começou a analisar o processo de negócio comunicação. Para isso, aplicou a modelagem de processos, uma das fases do BPM, composta pelas análises AS-IS e TO-BE.

$\mathrm{Na}$ análise AS-IS, também conhecida como modelagem do estado atual do processo, o objetivo precípuo é entender e descrever como um processo de negócio é realizado na organização Baldam et at (2007). Neste estudo de caso o processo de negócio comunicação. Procura-se, nessa modelagem tirar uma fotografia do processo de negócio.

O objetivo da análise TO-BE é modelar o estado desejado do processo. Destina-se a construir o processo de negócio que proporcionará inovação e permitirá a organização conceber, desenvolver e entregar produtos e serviços com os valores definidos nos objetivos de negócio e estratégicos.

A modelagem TO-BE apontou que a linha de negócio comunicação seria pautada 367 
em três processos de negócio: (i) e-Carta, (ii) e-FAC e (iii) e-Marketing Direto. O processo de negócio e-Carta é o foco deste artigo.

A partir da observação do participante, uma técnica de coleta de dados da modalidade de pesquisa estudo de caso, percebeu-se que a orientação por processos, que norteou 0 desenvolvimento do processo de negócio eCarta, foi muito além das modelagens AS-IS e TO-BE. Esse trabalho produziu os seguintes resultados:

- Conhecimento organizacional: todas as fases geraram muito conhecimento organizacional. Por exemplo, as fases de análise do estado atual e do estado desejado levaram à inovação do processo de negócio comunicação, que proporcionou a criação do serviço eCarta.

- Compartilhamento de conhecimento: Os modelos de processos de negócio gerados nas fases TO-BE e AS-IS foram armazenados em uma base de dados processual e compartilhados com toda a ECT. Logo, a modelagem de outros processos de negócio, entre eles o e-FAC e o e-Marketing Direto, não partiram do zero. Reutilizaram tais modelos.

- Aplicação de conhecimento: o conhecimento produzido em todas as fases do desenvolvimento do processo de negócio e-Carta (serviço e-Carta) foi a base para: (i) o desenvolvimento

da solução de TIC que sustenta o serviço e-Carta, (ii) a elaboração de programas de capacitação profissional, (iii) a construção da estrutura organizacional de gestão e operação do serviço e (iv) a definição do próprio modelo de gestão.

- Avaliação de conhecimento: a análise AS-IS permitiu a racionalização de atividades e recursos, o que levou a atualização do conjunto de conhecimentos críticos aos negócios da linha de comunicação.

- Integração organizacional: a modelagem (AS-IS e TO-BE) facilitou a identificação das interfaces (integração) com outros processos de negócio e sistemas de informação corporativos. Isso permitiu a integração (complementação) de conhecimento organizacional. Por exemplo, não foi necessário desenvolver um sistema de faturamento para o serviço e-Carta, pois a ECT já disponibiliza essas competências, pessoal e de tecnologia da informação, em uma solução de Enterprise Resource Planning - ERP. Essa intensa mobilização de conhecimento despertou a atenção de um dos componentes do GT que iniciara o curso de doutoramento em 2011, no Programa de Engenharia e Gestão do Conhecimento da Universidade Federal de Santa Catarina - 
UFSC, por intermédio do programa de bolsas de estudo da Universidade Corporativa dos Correios.

Assim, imediatamente ocorreu uma ligação dos resultados obtidos no desenvolvimento do processo de negócio eCarta com duas estratégias do plano estratégico da ECT, listadas a seguir:

- Desenvolver e implantar um modelo de GC.

- Desenvolver e implantar um modelo organizacional com base na orientação por processo.

A ideia é a seguinte: se os processos de conhecimento podem ser facilitados pela orientação por processo (ou o BPM), então é possível aplicar uma abordagem integrada dessas áreas de conhecimento, em vez de desenvolver modelos separados.

Com essa percepção inicial, procurouse identificar a relação entre GC e BPM a partir de uma fundamentação científica, conforme discutido na próxima seção.

\subsection{Fundamentação Teórica}

A revisão da literatura foi pautada na seguinte linha de pesquisa: integração entre GC e BPM. O objetivo era identificar, mediante a análise de alguns modelos de GC e BPM, relações de similaridades e complementaridades entre essas duas áreas, que fundamentasse a construção de um modelo de incorporação dos processos de conhecimento nas rotinas organizacionais para promover e facilitar a GC.
O Quadro 1 sintetiza os modelos de GC analisados, que estão posicionados entre os principais frameworks (modelos) da literatura.

Quadro 1 - Modelos de GC Analisados.

\begin{tabular}{|c|c|c|c|}
\hline Modelo & Ciclo de Vida & $\begin{array}{l}\text { Orientação } \\
\text { Estratégica }\end{array}$ & Aceleradores \\
\hline $\begin{array}{l}\text { Asian } \\
\text { Productivity } \\
\text { Organization } \\
\text {-APO }\end{array}$ & $\begin{array}{l}\text { - Identificar } \\
\text { - Criar } \\
\text { - Armazenar } \\
\text { - Compartilhar } \\
\text { - Aplicar }\end{array}$ & $\begin{array}{l}\text { Missão } \\
\text { Visão }\end{array}$ & $\begin{array}{l}\text {-Pessoas } \\
\text {-Processos } \\
\text {-Tecnologia } \\
\text {-Liderança }\end{array}$ \\
\hline GPO-WM & $\begin{array}{l}\text { Criar } \\
\text { Armazenar } \\
\text { Compartilhar } \\
\text { - Aplicar }\end{array}$ & $\begin{array}{l}\text { Negócio e } \\
\text { Processo } \\
\text { de Negócio }\end{array}$ & \begin{tabular}{|l}
-Cultura \\
-Processos \\
- Tecnologia \\
-Liderança
\end{tabular} \\
\hline $\begin{array}{l}\text { Administraçã } \\
\text { o Pública } \\
\text { Brasileira - } \\
\text { APB }\end{array}$ & $\begin{array}{l}\text { Identificar } \\
\text { Criar } \\
\text { Armazenar } \\
\text { - Compartilhar } \\
\text { Aplicar }\end{array}$ & $\begin{array}{l}\text { Plano } \\
\text { Estratégico }\end{array}$ & $\begin{array}{l}\text {-Cultura } \\
\text {-Processos } \\
\text { - Tecnologia } \\
\text {-Liderança }\end{array}$ \\
\hline
\end{tabular}

Fonte: (Nair, 2009, Heisig, 2009 e Batista, 2012).

Por sua vez, o Quadro 2 fornece uma visão dos modelos de BPM analisados e que estão entre os mais maduros apresentados na literatura sobre o assunto.

Quadro 2 - Modelos de BPM Analisados.

\begin{tabular}{|c|c|c|}
\hline Modelo & $\begin{array}{l}\text { Ciclo de } \\
\text { Vida }\end{array}$ & Foco \\
\hline Baldam & $\begin{array}{l}\text { - Planejamento } \\
\text { - Modelagem } \\
\text { - Execução } \\
\text { - Controle e análise }\end{array}$ & $\begin{array}{l}\text { Melhoria de } \\
\text { processos }\end{array}$ \\
\hline Jung & $\begin{array}{l}\text { - Criação } \\
\text { - Modelagem } \\
\text { - Pré-análise } \\
\text { - Execução } \\
\text { - Pós-análise } \\
\text { - Evolução } \\
\end{array}$ & $\begin{array}{l}\text { Integração entre } \\
\text { GC e BPM }\end{array}$ \\
\hline Houy & $\begin{array}{l}\text { - Desenvolvimento } \\
\text { da estratégia } \\
\text { - Modelagem } \\
\text { - Execução } \\
\text { - Monitoramento e } \\
\text { Controle } \\
\text { - Otimização } \\
\text { Melhoria }\end{array}$ & $\begin{array}{l}\text { Melhoria } \\
\text { processos }\end{array}$ \\
\hline
\end{tabular}

Fonte: (Baldam et. al, 2007; Jung, Choi e Song, 2007 e Houy, Fettke e Loos 2010). 


\subsection{Construção do Modelo de Incorporação dos Processos de Conhecimento}

Com base no trabalho realizado no desenvolvimento do processo de negócio eCarta e na avaliação dos modelos de GC e de BPM oportunizada pela revisão da literatura, concebeu-se o modelo de incorporação dos processos de conhecimento nas rotinas organizacionais da Figura 2, que tem as seguintes características:

- Abordagem integrada: os ciclos de GC e BPM foram integrados, a partir dos Quadros 1 e 2. Essa integração promove a articulação e mobilização dos viabilizados, ou fatores de contextos, que mais influenciam a GC: pessoas, processos, tecnologia e liderança.

Figura 2 - Modelo de Incorporação dos Processos de Conhecimento.

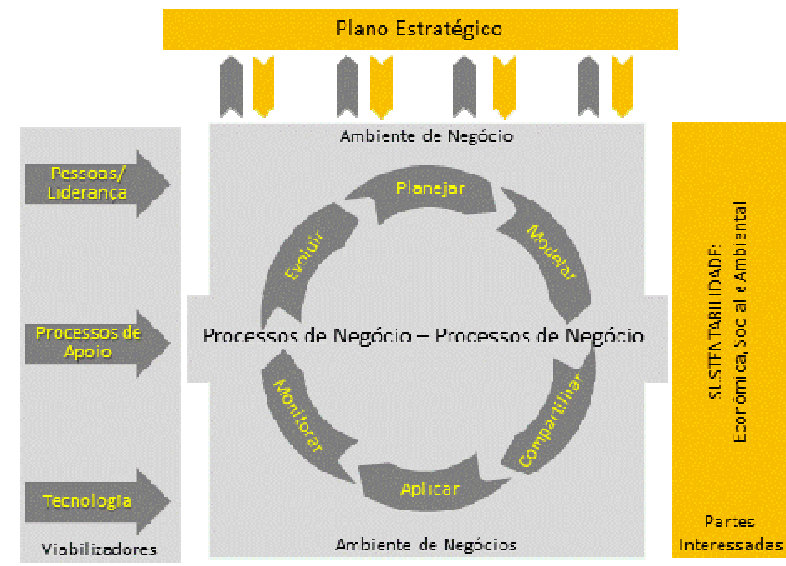

Fonte: Adaptado de Batista (2012).

- Planejamento: a referência principal do modelo proposto é o plano estratégico da organização. A finalidade maior dessa fase é identificar o conjunto de processos de negócio que levará à concepção, desenvolvimento e entrega dos produtos e serviços da organização e, por consequência, concretizarão os objetivos de negócio e estratégicos.

- Modelagem: essa é uma fase que proporciona intensa identificação e criação de conhecimento crítico à concretização os objetivos de negócio e estratégicos.

- Aplicação: a GC só faz sentido se ela adicionar valor aos produtos e serviços de uma organização e proporcionar inovação. A orientação por processos promove e facilita essas coisas.

- Monitoramento: monitorar e controlar os processos de negócio permite a avaliação dos conhecimentos, identificando aqueles que não mais contribuem para a concretização dos objetivos de negócio e estratégicos.

- Evolução: é uma fase crítica para a evolução da empresa, pois ela deve garantir que $\mathrm{GC}$, no seu processo final, realimente o plano estratégico da organização. Isso está indicado pelas setas em cor cinza na Figura 2.

- Foco: em negócios, buscando a sustentabilidade organizacional.

Além disso, o modelo proposto neste artigo foi concebido para promover e facilitar a incorporação dos processos de conhecimento nas rotinas de trabalho organizacionais, pois o ciclo da GC (planejar, modelar, compartilhar, aplicar, monitorar e 370 
evoluir) é aplicado aos processos de negócio, que são compostos pelas atividades críticas de negócio.

\subsection{Proposições}

A partir do modelo desenhado, este artigo formulou as seguintes proposições (P) de pesquisa:

- (P1): a modelagem AS-IS dos processos de negócio promove e facilita a identificação de conhecimentos críticos para a consecução dos objetivos de negócio e estratégicos.

- (P2): a modelagem TO-BE dos processos de negócio facilita a identificação de lacunas de conhecimentos críticos para a consecução dos objetivos de negócio e estratégicos.

- (P3): a modelagem TO-BE dos processos de negócio facilita a criação de conhecimentos críticos (socialização, explicitação, combinação e internalização) para a consecução dos objetivos de negócio e estratégicos.

- (P4): a modelagem TO-BE pode promover a inovação de processos, produtos e serviços.

- (P5): a modelagem TO-BE pode promover a criação de valor para clientes e mercados.

- (P6): as modelagens AS-IS e TO-BE facilitam a identificação da integração com outros processos de negócio e/ou sistemas corporativos (integração e complementação de conhecimentos).

- (P7): compartilhar modelos de processos de negócio promove $\mathrm{e}$ facilita a disseminação de conhecimentos críticos para a consecução dos objetivos de negócio e estratégicos.

- (P8): a integração entre GC e BPM facilita a incorporação dos processos de conhecimento nas rotinas de trabalho organizacionais.

- (P9): a integração entre GC e BPM facilita a mobilização de pessoas, processos de apoio, estruturas organizacionais, TI e métodos de trabalho (fatores de contexto de GC) com vista à realização dos objetivos de negócio e estratégicos.

- (P10): a execução dos processos de negócio representa a fase de aplicação de conhecimento.

- (P11): a execução de processos de negócio gera novos conhecimentos valiosos para a organização.

- (P12): o monitoramento e controle de processos de negócio facilitam a avaliação e evolução de conhecimentos organizacionais.

\subsection{Coleta de Dados}

A fim de verificar as proposições deste estudo de caso, foi realizada uma entrevista com os principais participantes do 
Cód. Benefícios da Orientação Por Processos (BPM) A modelagem AS-IS facilita a identificação dos

B1 conhecimentos críticos à realização dos objetivos de negócio e estratégicos

A modelagem TO-BE facilita a identificação de

B2 lacunas de conhecimentos críticos à realização dos objetivos de negócio e estratégicos

A modelagem TO-BE facilita a criação de

B3 conhecimentos críticos à realização dos objetivos estratégicos e de negócios

A modelagem TO-BE pode promover a inovação de

B4 processos, produtos e serviços, pois a visão por processos permite olhar facilmente para além das fronteiras da organização

B5 A modelagem TO-BE pode promover a criação de valor para clientes e mercados

As modelagens AS-IS e TO-BE facilitam a

B6 identificação da integração com outros processos de negócio e/ou sistemas corporativos (integração e complementação de conhecimentos)

Os modelos de processos de negócio gerados a partir das modelagens AS-IS e TO-BE são formas

B7 adequadas de armazenamento de conhecimentos críticos à realização dos objetivos de negócios e estratégicos

Os modelos de processos de negócio gerados a partir das modelagens AS-IS e TO-BE podem

B8 facilitar o compartilhamento dos conhecimentos críticos à realização dos objetivos de negócios e estratégicos

Os modelos de processos de negócio gerados a partir das modelagens AS-IS e TO-BE facilitam a disseminação dos conhecimentos necessários à realização dos objetivos estratégicos e de negócios

A integração entre GC e BPM facilita a

B10 incorporação dos processos de conhecimento (identificação, criação, compartilhamento, aplicação e avaliação) nas rotinas de trabalho organizacionais

A integração entre GC e BPM facilita a mobilização de pessoas, processos de apoio, estruturas

B11 organizacionais, TI e métodos de trabalho (fatores de contexto de GC) com vista à realização dos objetivos de negócio e estratégicos

B12 A execução de processos de negócio gera novos conhecimentos valiosos para a Empresa

A execução dos processos de negócio facilita a

B13 identificação dos benefícios da GC, uma vez que nem sempre é fácil mensurar os resultados práticos da aplicação de conhecimento organizacional

O monitoramento e o controle de processos de B14 negócio facilitam a avaliação e evolução dos conhecimentos críticos à realização dos objetivos de negócio e estratégicos

desenvolvimento do processo de negócio e-

Carta. Essa entrevista consistiu em apresentar aos entrevistados catorze benefícios (B) que

podem advir da orientação por processos
(Quadro 3). Para cada benefício o entrevistado deveria avaliá-lo de acordo com a seguinte escala:

- $[0]$ - Não ocorre.

- $\quad[1]$ - Ocorre em parte.

- [2] - Ocorre.

O Quadro 4 ilustra um exemplo de benefício apresentado aos participantes.

Quadro 3 - Lista Completa dos Benefícios Avaliados e Apresentados no Gráfico 1.

Fonte: Autoria Própria.

\section{Resultados}

O Gráfico 1 sintetiza os resultados da pesquisa, que contou com a participação de sete pessoas-chave. Entre eles o gestor do processo e-Carta e especialistas em serviços de comunicação empresarial.

Quadro 4 - Exemplo de Benefício Abordado na Entrevista.

\begin{tabular}{|c|l|c|}
\hline Cód. & \multicolumn{1}{|c|}{ Benefícios do BPM } & Marque \\
\hline $\mathrm{B}_{\mathbf{N}}$ & $\begin{array}{l}\text { Facilita a identificação de lacunas } \\
\text { de conhecimentos críticos à } \\
(\mathrm{N}=1-14)\end{array}$ & $\begin{array}{l}\text { realização dos objetivos de negócio } \\
\text { e estratégicos }\end{array}$ \\
\hline
\end{tabular}

Fonte: Autoria Própria.

Gráfico1 - Resultados da Entrevista.

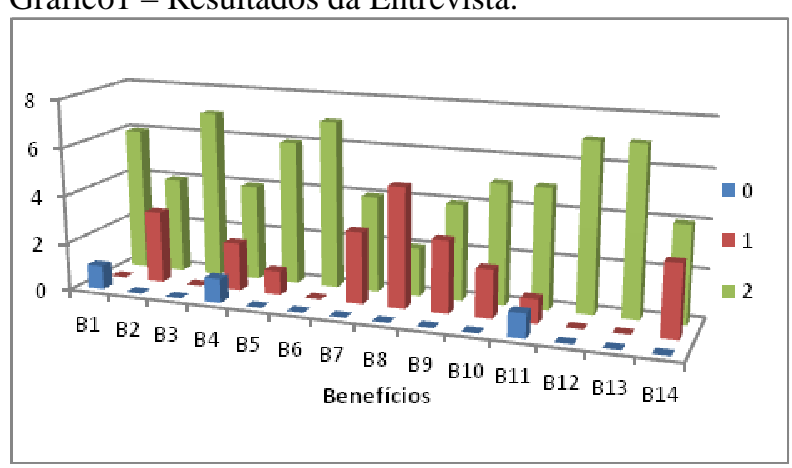

Fonte: Dados Primários.

As duas primeiras colunas do Quadro 5 faz uma correlação entre as proposições do artigo e o benefício correspondente avaliado. 
A terceira coluna, por sua vez, indica o grau de reforço das proposições a partir da avaliação dos benefícios realizada pelos entrevistados.

Quadro 5 - Correlação Entre Proposições e Benefícios.

\begin{tabular}{|c|c|c|}
\hline Proposição & Benefício & Grau de Reforço \\
\hline P1 & B1 & Alto \\
\hline P2 & B2 & Alto \\
\hline P3 & B3 & Alto \\
\hline P4 & B4 & Alto \\
\hline P5 & B5 & Alto \\
\hline P6 & B6 & Alto \\
\hline \multirow{3}{*}{ P7 } & B7 & Alto \\
\cline { 2 - 3 } & B8 & Médio \\
\cline { 2 - 3 } & B9 & Alto \\
\hline P7 & B10 & Alto \\
\hline P8 & B11 & Alto \\
\hline P8 & B12 & Alto \\
\hline P9 & B13 & Alto \\
\hline P10 & B14 & Alto \\
\hline
\end{tabular}

Fonte: Dados Primários.

Apenas dois processos críticos da GC serão destacados neste artigo: a identificação e criação dos conhecimentos organizacionais críticos para os negócios de uma organização. Esses processos foram avaliados por meio dos benefícios B1 e B3, respectivamente. Conforme é possível perceber a partir do Gráfico I e do Quadro 5, as duas proposições foram reforçadas, considerando que os benefícios que as representam obtiveram grau de reforço alto dos entrevistados.

\section{Considerações Finais e Trabalhos Futuros}

Estudos científicos sobre as vantagens da aplicação integrada da GC com o BPM ainda são poucos, particularmente no Brasil. Mas a pesquisa com foco na integração dessas duas áreas de conhecimento está em expansão.

O modelo apresentado neste artigo está sendo aplicado ao ambiente de negócio de uma empresa pública de grande porte que atuam em mercados altamente concorrenciais e é objeto de pesquisa de uma tese de doutorado. Logo, novos artigos serão publicados abordando os resultados da aplicação do modelo aqui proposto.

\section{Referências Bibliográficas}

BALDAM, R. et al. Gerenciamento de processos de negócios. 1 ed. São Paulo: Érica, 2007.

BATISTA, F. F. Modelo de gestão do conhecimento para a administração pública brasileira. Rio de Janeiro: IPEA, 2012.

FRATANTONIO, W. A. Uma Discussão Sobre a Utilização do Estudo de Casos como Método de Pesquisa em Ciências Gerenciais. XXXII Encontro a ANPAD, 2008. Rio de Janeiro-RJ.

GIL, A. C. Como elaborar projetos de pesquisa. 5. São Paulo: Atals, 2010. 184

HEISIG, P. Harmonisation of knowledge management. Journal of Knowledge Management, v. 13, n. 4, p. 4-31, 2009.

HOUY, C.; FETTKE, P.; LOOS, P. Empirical research in business process managementanalysis of an emerging field of research. Business Process Management Journal, v. 16, n. 4, p. 619-661, 2010.

JUNG, J. S.; CHOI, I.; SONG, M. S. An integration architecture for knowledge management systems and business process management systems. Computers in Industry, v. 58, n. 1, p. 21-34, Jan 2007.

MARCONI, M. D. A.; LAKATOS, E. M. 
Fundamentos de metodologia científica. 5 . São Paulo: Atlas, 2003.

MARTINS, G. A. Estudo de Caso. 2. São

Paulo: Atlas, 2008.

NAIR, P. P., K. Knoledge Management:

Facilitators' Guide. Tokyo: APO, 2009.

SEIDEL, S. A Business Process Management Perspective on Creativity: Creativity Intensive Processes. AMCIS, 2007.

SOUZA, L. L. C. D. Mecanismos de Coordenação e Práticas da Gestão do Conhecimento na Rede de Valor Terceirizada [tese]. 2011. Departamento de Engenharia e Gestão do Conhecimento, UFSC.

VENTURA, M. M. O estudo de caso como modalidade de pesquisa. Revista SoCERJ, v. 20, n. 5, p. 383-386, 2007.

YIN, R. K. Estudo de Caso: Planejamento e Métodos. 4 ed. Porto Alegre: 2010. 